\section{(2) OPEN ACCESS}

\title{
Development and evaluation of a novel protective device for upper gastrointestinal endoscopy in the COVID-19 pandemic: the EBOX
}

\author{
Robert William John Mcleod (D) , ${ }^{1,2}$ Neil Warren, ${ }^{3}$ Stuart Ashley Roberts ${ }^{2}$
}

\begin{abstract}
${ }^{1}$ School of Psychology, Cardiff University, Cardiff, UK

${ }^{2}$ Radiology Department, Cardiff and Vale University Health Board, Cardiff, UK

${ }^{3}$ WIMAT, Cardiff University, Cardiff, UK
\end{abstract}

\section{Correspondence to}

Dr Robert William John Mcleod, Cardiff University School of

Psychology, Cardiff CF10 3AS,

UK; mcleodrwj@googlemail.com

Received 14 May 2020

Revised 28 May 2020

Accepted 7 June 2020

Published Online First

12 June 2020

\section{Check for updates}

(C) Author(s) (or their employer(s)) 2021. Re-use permitted under CC BY-NC. No commercial reuse. See rights and permissions. Published by BMJ.

To cite: Mcleod RWJ,

Warren N, Roberts SA.

Frontline Gastroenterology

2021;12:188-192.

\begin{abstract}
Background During the COVID-19 pandemic, aerosol-generating procedures such as upper gastrointestinal endoscopy (UGIE) have been considered high risk. We designed a novel acrylic box (endoscopy box (EBOX)) with the intention of limiting aerosol and droplet spread during such procedures. We evaluated clinical utility, impact on the endoscopy team and also assessed the impact of the EBOX on macroscopic droplet spread from a simulated cough during UGIE.
\end{abstract}

Methods Clinical utility was evaluated prospectively via EBOX use on 15 patients undergoing endoscopic retrograde cholangiopancreatography (13) or endoscopic ultrasound (2). Feedback was recorded from the endoscopy team regarding ease of positioning, impact of the EBOX on procedural performance and cleaning. A cough was simulated via explosion of a hyperinflated balloon containing $0.75 \mathrm{~mL}$ of ultraviolet disclosing lotion within the oral cavity of a mannequin, with and without the EBOX. Macroscopic spread was then evaluated with a ultraviolet torch.

Results Three endoscopists and the team members found that the EBOX did not hamper the procedure and felt it was a useful adjunct to full personal protective equipment (PPE). Simulated cough without the EBOX identified macroscopic spread up to $2.3 \mathrm{~m}$ away from the patient's mouth as well as onto key areas such as the exposed neck of the endoscopist, which is not considered in current PPE guidance. Simulated cough using the EBOX significantly reduced macroscopic spread onto key areas of the healthcare workers.

Conclusions The EBOX is a valuable adjunct to recommended PPE for UGIE, but still allows these procedures to be performed in the standard manner.

\section{Significance of this study}

What is already known about this subject?

- Since the COVID-19 pandemic, physical barriers to potentially reduce droplet and aerosol spread during endotrachea intubation have been described.

What this study adds?

- To our knowledge, this is the first physical barrier described for use in upper gastrointestinal endoscopy, and we have demonstrated its efficacy in vitro, and its clinical utility in a small case series.

How might it impact on clinical practice in the foreseeable future?

- Use of such a device may become routine in upper gastrointestinal endoscopy to reduce the risk of nosocomial infection, but further studies on both generalisability and efficacy are required.

\section{INTRODUCTION}

During the COVID-19 pandemic, many healthcare workers (HCW) have lost their lives, some of which is no doubt related to direct patient contact. ${ }^{12}$ Protection of healthcare staff remains of paramount importance in combating the crisis. Interventional medical procedures that have the potential to generate droplet and aerosols have the potential to put HCWs at particularly high risk. ${ }^{34}$

Upper gastrointestinal endoscopy (UGIE) including endoscopic retrograde cholangiopancreatography (ERCP) and endoscopic ultrasound (EUS) is considered to be an aerosol-generating procedure (AGP), and current guidance requires full personal protective equipment (PPE) with as a minimum an FFP3 or N95 
mask. ${ }^{5}$ Other protective equipment is also required including a full surgical gown covering the arms, torso and thighs, a plastic apron, face visor and gloves. The latter protect against droplets generated during intubation and extubation, particularly from retching and coughing. $^{78}$

The extent of droplet spread onto the PPE and elsewhere is not known during UGIE but it is established that doffing errors can occur leading to selfcontamination. ${ }^{9}$ Droplet spread is likely to occur at various stages of UGIE but particularly, during intubation and extubation.

The authors became aware of the intubationbox. com project in the USA (personal communication, Prof Trisha Greenhalgh, CEBM Oxford), which has subsequently been published. ${ }^{10}$ Embedded in the website was a video of an experiment to simulate a cough during endotracheal intubation, demonstrating the effectiveness of a physical barrier. ${ }^{11}$ We had a box built (DIPEC Plastics, Cardiff) to the exact specification on the website. Feedback from the anaesthetists was highly variable, but the majority were concerned with the relatively limited access and instability of the box on the bed or trolley.

With this in mind, our aim was to develop a physical barrier to reduce the amount of droplet spread onto the PPE of HCW during UGIE. Taking the stability and access issues into consideration, a new prototype box was designed and built with practical clinical utility in mind for UGIE procedures. Following feedback from endoscopy nursing staff and radiographic staff, a second prototype was built-The endoscopy box (EBOX). The EBOX, although open on its underside, has inward facing flanges which allow a sheet of acrylic to be inserted after placement. This sits over the flange but under the patient's head thus stabilising the box (preventing instability issues of the previous design). There are access ports cut into the acrylic box at the head end for the assistant and on the side for the endoscopist.

The purpose of this study is to:
- Describe a new device (EBOX) for UGIE which may protect healthcare workers during the COVID-19 pandemic.

- Evaluate the effectiveness of the EBOX in containing droplets during a simulated cough, while evaluating the effectiveness of current PPE guidance with and without the EBOX.

- Evaluate the clinical utility of the EBOX in a small series of complex endoscopy procedures-ERCP and EUS.

\section{METHODS}

We gained approval from the Medical Director of the Clinical Innovation Partnership between Cardiff University and Cardiff and Vale University Health Board, and the Executive Medical Director of Cardiff and Vale University Health Board to allow us to pursue this project with urgency during the COVID-19 pandemic. We sought and followed the advice provided by the Infection Prevention and Control Department at the University Hospital of Wales regarding safe cleaning and storage of the EBOX.

\section{Clinical utility}

The EBOX prototype is seen in figure $1 \mathrm{~A}$ without application of disposable drapes, figure 1B with draping of the assistants access window continuing to the cranial aspect of the endoscopist's access and figure 1C with draping of the caudal end of the EBOX, containing the patient's torso and continuing to the caudal aspect of the endoscopist's access (3M Steri-Drape Fluoroscope Drape, 3M Medical Solutions Division, UK). This creates an inner pair of curtains at the endoscopist's access window. When the patient and the drapes are in the procedural position, a final drape is placed over this window and an access hole cut, to accommodate the endoscopist's right arm for intubation and extubation. Detailed information regarding our current recommendations for set-up, draping and cleaning can be found at www.ebox.wales.

All clinical procedures in the study were performed between the 20 April and 7 May 2020. Following

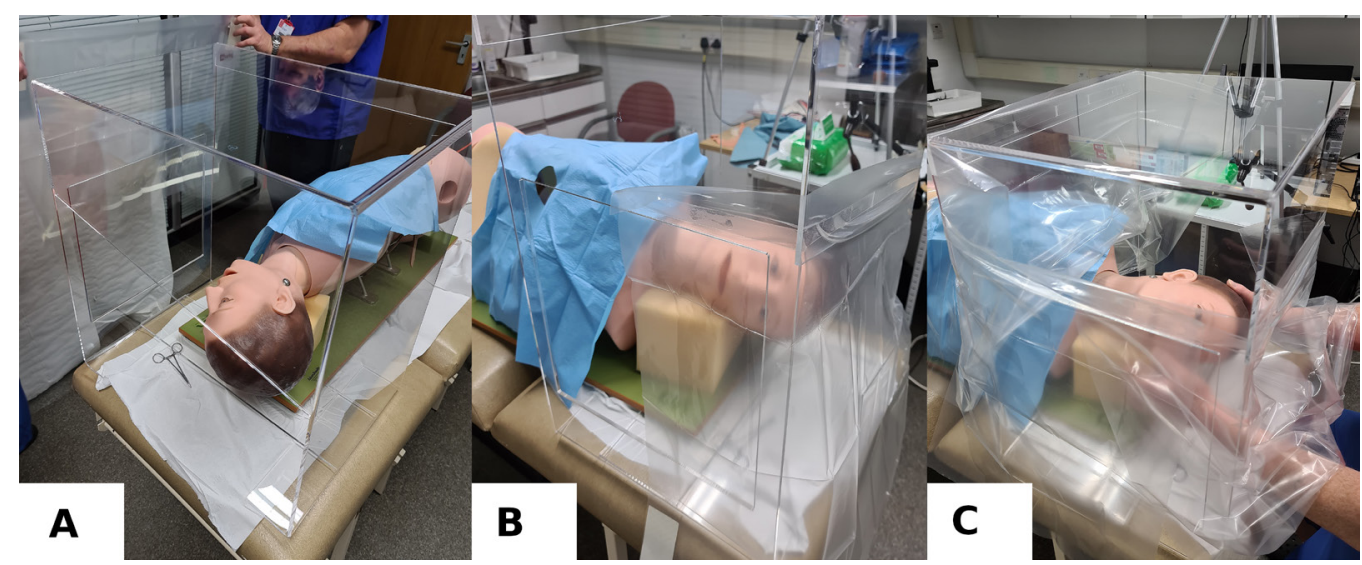

Figure 1 Simulated patient with EBOX in place (A), with the cranial drapes in place (B) and with the side operators drapes in place (C). 
prior verbal consent from 15 patients (13 ERCP, 2 EUS), the box was placed in position and the procedures performed in the usual fashion. Care was taken to cover the biopsy access channel with a swab, during guidewire exchange. At the end of each procedure, the EBOX was placed on the floor of the room, and the whole room was left fallow for 1 hour according to local protocol. The EBOX was subsequently cleaned; the drapes were rolled with the contaminated side on the inside before removal and the EBOX cleaned with Clinell wipes for visible contamination, followed by Actichlor Plus solution (Ecolab, UK) at $10000 \mathrm{ppm}$ according to local protocols.

Verbal feedback from the patients and endoscopy nursing staff for all procedures, and radiographic staff for ERCP, was then obtained. Further feedback was obtained from another two endoscopists who used the EBOX for three cases.

\section{Simulated cough experiment}

The laboratory study was performed on 27 April 2020. An endoscopy training mannequin (AC4, OGI Phantom, Adam, Rouilly, UK) was positioned in the usual left lateral position for UGIE. A cholangiogram catheter with a Luer lock at the caudal end (Taut, Teleflex Medical Europe) was fitted with a balloon at the open cranial end containing $0.75 \mathrm{~mL}$ of UV disclosing lotion (GlitterBug Potion, Brevis Corporation, USA). Using biopsy forceps passed retrogradely up the oesophagus, the catheter was backloaded into the pharynx of the mannequin, followed by visual confirmation of the correct position with white and UV light. A $50 \mathrm{~mL}$ syringe containing air was attached to the Luer lock and inflated until the balloon burst, crudely simulating a cough. This was performed with the endoscopist and airway assistant wearing the recommended PPE as described in the introduction with the addition of a surgical hat. Prior to any simulated cough, both operators were assessed with UV light in a darkened room for any unexpected contamination by UV disclosing solution.

The simulated cough was then performed with and without the EBOX in place, with both operators' arms inside the EBOX in the usual UGIE position. The spread of droplets following the balloon burst was illuminated with UV light in a darkened room to assess the distribution of the UV disclosing lotion.

\section{RESULTS}

\section{Clinical utility}

The box was stable in all of the procedures undertaken with good patient acceptability on an X-ray fluoroscopy table for ERCP and a standard trolley for EUS. The patients were reassured by positioning them first without the EBOX, then sliding the EBOX in place before applying the drapes. Drape application took less than $1 \mathrm{~min}$ by the final case in the series, as the technique evolved. The endoscopist and the airway

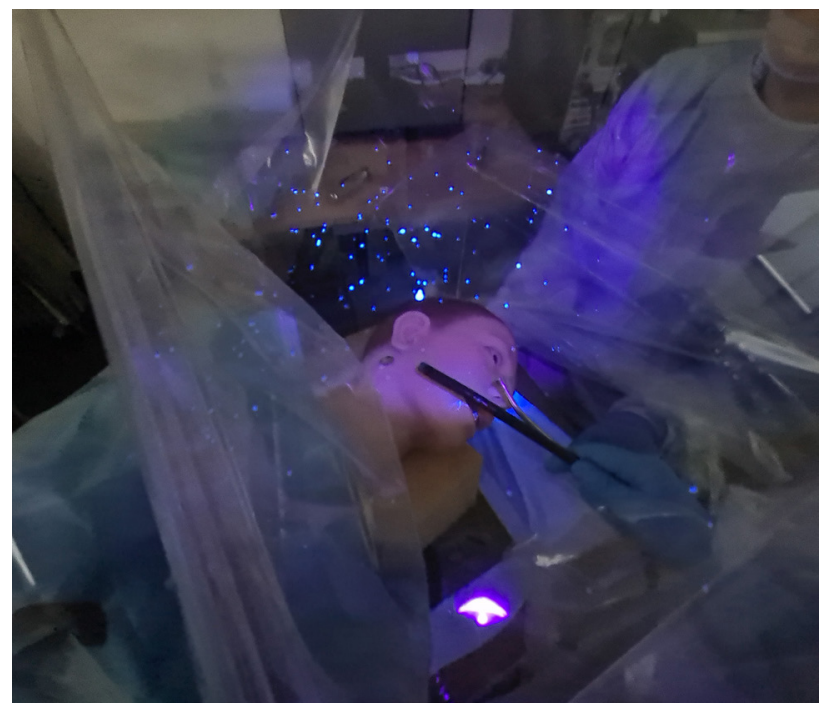

Figure 2 UV droplets visible on the inside roof of endoscopy box.

assistant found no impairment to standard practice. Fluoroscopy images during ERCP could be obtained, although careful repositioning of the EBOX was required to ensure the top of the liver could be encompassed in the field of view. We rotated the image intensifier from portrait to landscape, to allow better positioning of the intensifier during ERCP.

All patients received conscious sedation (Midazolam and Fentanyl), delivered via an extension tube exiting the caudal end of the EBOX. Patient tolerance of the procedure was not impaired, and all cases were successfully completed. No patients described feeling claustrophobic while in the EBOX.

\section{Simulated cough experiment}

There was no prior contamination by UV disclosing solution on the EBOX and drapes, either operator or the surrounding environment.

\section{With the EBOX in position}

UV disclosing lotion was present on the internal surfaces of the EBOX and drapes particularly on the endoscopist's side but also on the airway assistant's side and the roof of the EBOX (figure 2).

UV disclosing lotion was also present on the right forearm and glove of the endoscopist, and the left forearm and glove of the airway assistant which were inside the EBOX. No UV disclosing lotion was seen on the left arm of the endoscopist outside the EBOX, or the right arm of the airway assistant inside the EBOX but behind the head.

No UV disclosing lotion was seen on the upper arms, torso, visor, head cover or mask of either operator. A small amount of UV disclosing lotion was seen on the lower aspect of the gown of both operators, presumably via the inferior aspect of the drapes which were not fixed. No UV disclosing lotion was seen on unprotected skin, in particular, the neck of the endoscopist 


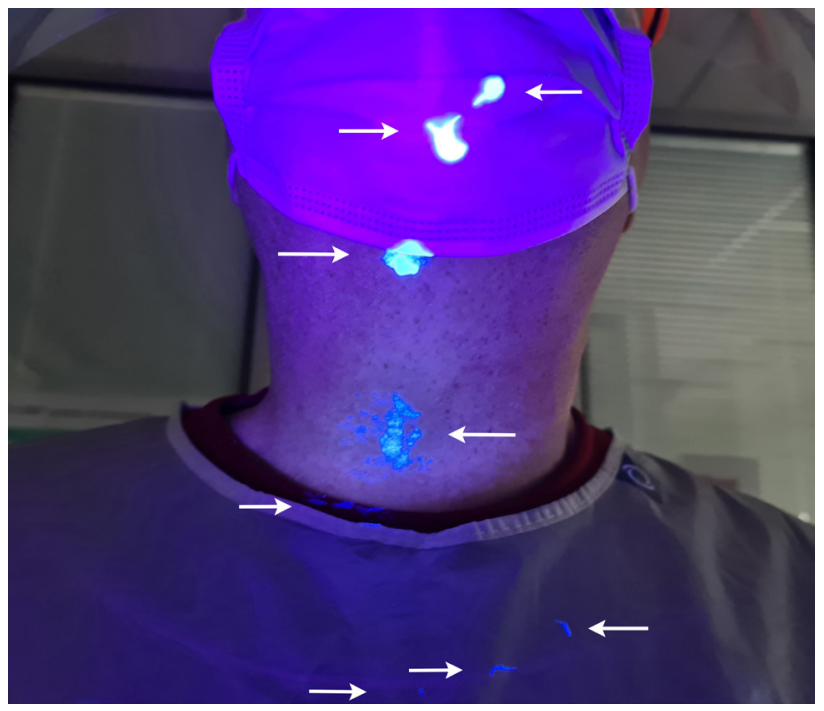

Figure 3 UV droplet contamination over gown, exposed neck and under visor onto facemask following simulated cough without endoscopy box (arrows highlight macroscopic contamination).

and airway assistant. No UV disclosing lotion was visible in the local environment.

\section{Without the EBOX in position}

Following the simulated cough, UV disclosing lotion was present on the forearms, upper arms, upper and lower torso, and visor of both operators. It was present on both gloves of the endoscopist, the control wheels of the endoscope and the left glove of the airway assistant. There was a significant volume of UV disclosing lotion on the uncovered neck of the endoscopist, under the full face visor onto the face mask (figure 3). There was no UV disclosing lotion on the head of either operator. Further UV disclosing lotion was seen in the surrounding environment up to $2.3 \mathrm{~m}$ away.

\section{DISCUSSION}

This study has demonstrated two major findings; the EBOX is practical for clinical use, and is effective in significantly containing macroscopic droplet spread during a simulated cough.

In order to maximise clinical utility in the EBOX design, we noted the limitations described by Canelli et $a l$, akin to the feedback received from our anaesthetic colleagues, and aimed to surpass these limitations. ${ }^{10}$ Subsequently, others have described further limitations but fortunately we had already addressed those which may be relevant to UGIE. ${ }^{12}$ We improved access by using plastic drapes over wide access ports in the acrylic, which allow greater freedom of movement by the operator when compared with small fixed circular holes for access. Stability of the EBOX was addressed by using the weight of the patient in the design as they rest on a support. This did not impair rapid removal of the box if there were an adverse event such as cardiac arrest. We rehearsed this with a volunteer in the EBOX and it could be removed in less than a second. Fortunately, no such adverse events occurred in this small series.

We found that there was significant macroscopic droplet spread onto the endoscopist's neck without the EBOX in place. At the point of simulated cough, the endoscopist was looking at an endoscopy monitor, in keeping with standard practice; therefore, the neck was slightly extended. This likely explains the distribution of the UV disclosing lotion. Neck protection is not included in current Public Health England (PHE) PPE guidance for AGPs. ${ }^{6}$ If correct doffing procedures are followed to the letter, contamination on the HCW could remain. No neck protection or washing protocols are in place in any guidance, as far as we know.

The EBOX did not completely abolish macroscopic droplet spread outside the box. UV disclosing lotion was seen on the lower gown of both operators with the EBOX in place, presumably due to the lack of fixation inferiorly. As a consequence, we have now modified our protocol to address this by fixing the lower drapes. We also believe that the cough being partially contained will cause a loss of momentum in the cough particle cloud, reducing distance travelled and potentially the likelihood of any particle from settling. ${ }^{13}$

As we move into the deceleration and recovery phase of the pandemic, it is likely that more UGIE will be performed with anaesthetic throat spray only. From our experience, patients tend to gag and cough more in this situation. This may enhance the value of the box in protecting HCW. All of our procedures were performed with conscious sedation and all tolerated the presence of the EBOX well. Although further work on patient tolerance is required with UGIE using throat spray, the EBOX was in position and fully draped prior to delivery of sedation. Further studies are needed to evaluate generalisability, and these are in development in Wales.

There are limitations to this study: the lack of generalisability and the fact this remains a simulated cough but we are addressing the former, and further studies are required to evaluate the full efficacy. In the simulated cough experiment, we used a significantly smaller volume than previously described $(0.75 \mathrm{~mL}$ of UV disclosing solution compared with $10 \mathrm{~mL}$ used by Canelli et al) in an effort to more realistically simulate a cough. ${ }^{10}$

While the findings from studies in Newcastle in the UK and Italy are encouraging for both the HCW community generally, ${ }^{14}$ and endoscopy specifically, ${ }^{15}$ the authors in these studies also recognise the limitations of their findings. COVID-19 is indiscriminate, and if this device saves the life of even one HCW, use of the EBOX is justified, particularly as it costs so little to produce $(£ 60)$. It is reusable, and the cost of the disposable drapes per case is approximately $£ 6$. While the authors consider that the EBOX has significant potential clinical utility, it is important to stress that 
this is an adjunct to full PPE for AGPs. Such PPE was worn by all $\mathrm{HCW}$ in the procedure room during the evaluation of clinical utility according to current PHE guidance. We feel that one of the primary benefits of the EBOX is the reduction in droplet load and limiting macroscopic droplet transfer onto key areas such as exposed areas of the head and neck, where doffing errors often take place. ${ }^{9}$

In conclusion, we have demonstrated the EBOX is a useful adjunct to full PPE for UGIE. It does not impair performance of the procedures, costs little and this study has exposed concerns regarding droplet spread onto the endoscopist which are not addressed by current PPE guidance for UGIE. We recognise that further evaluation work is required including the following:

- Evaluate generalisability of the EBOX in wider endoscopic practice by user feedback.

- Evaluate the role of the EBOX in containing and removing aerosol-generated particles as well as droplets.

- Evaluate overall efficacy of the EBOX in reducing viral transmission rates to HCWs.

However, the publication of the latest British Society of Gastroenterology guidance for the deceleration and recovery phase of the pandemic ${ }^{16}$ will increase the number of UGIE procedures, and the EBOX may have a valuable role to play.

Acknowledgements Nursing and radiographic colleagues Radiology and Endoscopy at UHW. Jason Callow at DIPEC Plastics, Cardiff. Trisha Greenhalgh, CEBM Oxford. Intubation Box Charitable Trust and all the donors.

Contributors All authors performed experiments. SAR and RWJM wrote the manuscript. SAR performed 12 of the 15 clinical studies. Literature search: all authors. Data analysis and interpretation: all authors.

Funding The authors have not declared a specific grant for this research from any funding agency in the public, commercial or not-for-profit sectors.

Disclaimer The authors have no financial interest in the production of the EBOX. All of the funds raised by the Intubation Box Charitable Trust are spent on providing the EBOX to users, as well as further research and development.

Competing interests None declared.

Patient consent for publication Obtained.

Provenance and peer review Not commissioned; externally peer reviewed.

Data availability statement Data are available upon reasonable request.

Open access This is an open access article distributed in accordance with the Creative Commons Attribution Non Commercial (CC BY-NC 4.0) license, which permits others to distribute, remix, adapt, build upon this work noncommercially, and license their derivative works on different terms, provided the original work is properly cited, appropriate credit is given, any changes made indicated, and the use is non- commercial. See: http://creativecommons.org/licenses/by-nc/4. $0 /$.

\section{ORCID ID}

Robert William John Mcleod http://orcid.org/0000-0003-32216896

\section{REFERENCES}

1 Belingheri M, Paladino ME, Riva MA. Beyond the assistance: additional exposure situations to COVID-19 for healthcare workers. J Hosp Infect 2020. doi:10.1016/j.jhin.2020.03.033. [Epub ahead of print: 31 Mar 2020].

2 Wang J, Zhou M, Liu F. Reasons for healthcare workers becoming infected with novel coronavirus disease 2019 (COVID-19) in China. J Hosp Infect 2020;105:100-1.

3 Tran K, Cimon K, Severn M, et al. Aerosol generating procedures and risk of transmission of acute respiratory infections to healthcare workers: a systematic review. PLoS One 2012;7:e35797.

4 WHO. Modes of transmission of virus causing COVID-19: implications for IPC precaution recommendations. Sci Br 2020;29:1-3.

5 British Society of Gastroenterology. COVID-19: Bsg and BASL advice for healthcare professionals in gastroenterology and hepatology, 2020. Available: https://www.bsg.org.uk/ covid-19-advice/bsg-and-basl-covid-19-advice-for-healthcareprofessionals-in-gastroenterology-and-hepatology/ [Accessed 2 May 2020].

6 Public Health England. COVID-19: personal protective equipment use for aerosol generating procedures - GOV, 2020. Available: https://www.gov.uk/government/publications/covid19-personal-protective-equipment-use-for-aerosol-generatingprocedures [Accessed 2 May 2020].

7 Ferioli M, Cisternino C, Leo V, et al. Protecting healthcare workers from SARS-CoV-2 infection: practical indications. Eur Respir Rev 2020;29:200068-10.

8 Wax RS, Christian MD. Practical recommendations for critical care and anesthesiology teams caring for novel coronavirus (2019-nCoV) patients. Can J Anaesth 2020;67:568-76.

9 Okamoto K, Rhee Y, Schoeny M, et al. Impact of doffing errors on healthcare worker self-contamination when caring for patients on contact precautions. Infect Control Hosp Epidemiol 2019;40:559-65.

10 Canelli R, Connor CW, Gonzalez M, et al. Barrier Enclosure during endotracheal intubation. $N$ Engl J Med Overseas Ed 2020;382:1957-8.

11 Intubation Box - Aerosol Box a Protective Shield Against Covid-19. Available: https://intubationbox.com/ [Accessed 2 May 2020].

12 Rosenblatt WH, Sherman JD. More on barrier Enclosure during endotracheal intubation. N Engl J Med 2020;382:e69.

13 Bourouiba L. Turbulent gas clouds and respiratory pathogen emissions: potential implications for reducing transmission of COVID-19. JAMA 2020:E1-2.

14 Hunter E, Price DA, Murphy E, et al. Correspondence first experience of health-care workers in England. Lancet 2020;6736:19-20.

15 Repici A, Aragona G, Cengia G, et al. Low risk of covid-19 transmission in Gi endoscopy. Gut;25:gutjnl-2020-321341.

16 BSG. BSG Guidance on recommencing GI Endoscopy in the deceleration \& early recovery phases of the COVID-19 pandemic, 2020. Available: https://www.bsg.org.uk/covid-19advice/bsg-guidance-on-recommencing-gi-endoscopy-in-thedeceleration-early-recovery-phases-of-the-covid-19-pandemic [Accessed 2 May 2020]. 